\title{
The Evolving Biology of Microglia in Alzheimer's Disease
}

\author{
Tarja M. Malm • Taylor R. Jay • Gary E. Landreth
}

Published online: 18 November 2014

(C) The American Society for Experimental NeuroTherapeutics, Inc. 2014

\begin{abstract}
Alzheimer's disease (AD) is typified by a robust microglial-mediated inflammatory response within the brain. Indeed, microglial accumulation around plaques in $\mathrm{AD}$ is one of the classical hallmarks of the disease pathology. Although microglia have the capacity to remove $\beta$-amyloid deposits and alleviate disease pathology, they fail to do so. Instead, they become chronically activated and promote inflammationmediated impairment of cognition and cytotoxicity. However, if microglial function could be altered to engage their phagocytic response, promote their tissue maintenance functions, and prevent release of factors that promote tissue damage, this could provide therapeutic benefit. This review is focused on the current knowledge of microglial homeostatic mechanisms in $\mathrm{AD}$, and mechanisms involved in the regulation of microglial phenotype in this context.
\end{abstract}

Keywords Alzheimer's disease · Microglia · Inflammation · Phagocytosis · Abeta

\section{Microglia are Important for Normal Brain Function}

Microglia account for approximately $5-12 \%$ of the total number of cells in the brain, depending on the anatomic region [1]. While microglial origins have historically been controversial, Alliot et al. [2] and Ginhoux et al. [3] demonstrated that microglia are not derived from progenitors in the bone marrow - a surprising

T. M. Malm $(\bowtie)$

A.I. Virtanen Institute for Molecular Sciences, University of Eastern

Finland, P.O. Box 1627, 70211 Kuopio, Finland

e-mail: tarja.malm@uef.fi

T. R. Jay $\cdot$ G. E. Landreth

Department of Neurosciences, Alzheimer Research Laboratory,

School of Medicine, Case Western Reserve University, Cleveland,

OH 44106, USA finding as it was thought that these cells had the same embryonic origins as other mononuclear phagocytes. Rather, they originate from primitive hematopoetic precursors in the yolk sac. These cells enter the central nervous system (CNS) over a brief interval around $\mathrm{E} 9.5$, and, at least under homeostatic conditions, are primarily self-renewing throughout the life of the organism.

Microglia also have unique functions in the maintenance of CNS homeostasis. Microglia in a healthy CNS are uniformly distributed throughout the brain where they display a ramified morphology characterized by long, thin, and elaborate processes, and a small cell soma. Although these microglia were often described as being in a resting or quiescent state, key studies using 2-photon imaging $[4,5]$ showed that microglia constantly survey the brain parenchyma by constantly extending and retracting their processes within a circumscribed domain of about $80 \mu^{3}$, and are more properly termed "surveillant". Importantly, these studies also demonstrated the ability of microglia to make contacts with other neighboring cells, such as astrocytes, neurons, and vascular endothelial cells. These contacts allow microglia to monitor synaptic function, which has been shown to be important for complement-mediated synaptic pruning in both development and following learning [6]. This constant surveillance also allows microglia to respond rapidly to injury. Microglia migrate to sites of injury in a manner dependent on adenosine triphosphate and initiate tissue repair functions $[5,7]$, including the phagocytic removal of dead cells and debris. They also directly interact with neurons in these settings, and have been reported to prune presynaptic boutons following ischemia [8].

\section{Microglia in the Alzheimer's Disease Brain}

The recognition of the significance of the microglial response in the Alzheimer's disease (AD) brain is owing largely to early studies, which showed accumulation of microglia around 
amyloid plaques and initial success using nonsteroidal antiinflammatory drugs to modulate AD pathology (reviewed by McGeer and Rogers [9]). Since these landmark studies, our understanding of microglial biology in the context of AD has advanced dramatically. However, we are still far from understanding the finely tuned responses of these cells in the diseased brain.

Microglia accumulate in the vicinity of dense-core plaques and a few are also found surrounding diffuse $\beta$-amyloid $(A \beta)$ deposits $[10,11]$. Microglia are converted into a proinflammatory, "activated" phenotype by exposure to extracellular soluble and fibrillar $A \beta$ [12], and through direct interactions with neurons that exhibit accumulation of intraneuronal $A \beta$ [13]. Once recruited to plaques $[14,15]$, microglia undergo morphologic changes, such as increased soma size and thickened processes that they invest into the plaques [15]. These changes are accompanied by production of various inflammatory mediators and changes in cell surface receptor expression.

The role of microglia in the progression of AD is controversial. It is generally appreciated that patients with $A D$ have increased levels of inflammatory cytokines [16]. Moreover, it has been argued that the microglia-derived cytokines are responsible for the neuronal death observed in the AD brain, though this linkage remains both unproven and controversial. Excessive production of proinflammatory mediators also increases $A \beta$ levels. Proinflammatory cytokines can increase $A \beta$ accumulation by promoting secretion of amyloid precursor protein (APP) and stimulating the amyloidogenic cleavage of APP by $\gamma$ - and $\beta$-secretase in astrocytes [17-19]. In addition, increased inflammation lowers the levels of $A \beta$ degrading enzymes, such as neprilysin and insulin-degrading enzyme [20], the expression of A $\beta$-binding receptors [21], and the phagocytic capacity of microglia $[22,23]$, thus reducing $A \beta$ clearance. Together, these phenomena offer potential explanations as to why microglia fail to prevent the accumulation of $A \beta$ in the diseased brain.

While the relative contribution of these different factors is still unknown, it is indisputable that microglia in the $\mathrm{AD}$ brain are unable to remove effectively fibrillar forms of amyloid from the brain, resulting in the progressive accumulation of extracellular, deposited forms of amyloid in the brain parenchyma. It is enigmatic that despite their inherent capacity to eliminate foreign material, they fail to clear amyloid from the $\mathrm{AD}$ brain. This is not due to a generalized impairment of the phagocytic response, as microglia in the $\mathrm{AD}$ brain can readily phagocytose cargo that has been opsonized by antibodies, including amyloid [24, 25].

\section{Microglial Phenotypes}

Modulation of microglial activation to promote phagocytosis and limit proinflammatory cytokine release has been under intensive investigation as a potential treatment strategy to combat AD. Microglial activation states have been classically described as being either classically activated (M1) or alternatively activated (M2). These activation states have been characterized based on the cytokine secretion profile or alterations of cellular gene expression. Typically, M1-polarized microglia secrete an array of proinflammatory cytokines, such as tumor necrosis factor (TNF)- $\alpha$, interleukin (IL)-1, IL-6, IL12, IL-18, nitric oxide, and prostaglandins, and are relatively poor phagocytes. Conversely, M2-polarized microglia secrete anti-inflammatory cytokines, such as IL-4, IL-10, IL-13, and transforming growth factor- $\beta$, are highly potent phagocytes, and upregulate their expression of arginase 1, Fizz1, and Ym1. However, this current system for categorizing microglial phenotype arises from studies of peripheral macrophages, which fail to recapitulate fully microglial functions and is an oversimplification. Microglia do not conform to these binary phenotypes, but rather assume a broad range of functional roles that constantly change in response to the microenvironment, as reviewed by Gertig and Hanisch [26].

\section{Microglial Recruitment to Plaques}

When microglia encounter tissue damage or pathogens during their constant surveillance of the brain parenchyma, they are attracted to and undergo directed migration toward those sites of the damage. $A \beta$ itself can attract microglia, and this response is likely to reflect the interaction of $A \beta$ with both microglia and astrocytes, which are then stimulated to secrete chemokines $[27,28]$. The $\mathrm{C}-\mathrm{C}$ chemokine receptor type (CCR) 2-chemokine (C-C motif) ligand (CCL) 2 pathway has been shown to be especially important in mediating microglial recruitment to the plaques. Levels of CCL2 are increased in transgenic mouse models of $\mathrm{AD}$ and in the human AD brain [29-32]. Likewise, microglia in CCR2 knockout mice show impaired migration towards the site of $A \beta$ plaques and have higher $A \beta$ levels in the brain $[33,34]$. Once attracted to the plaque, microglia secrete chemokines, including chemokine (C-X-C motif) ligand 2, CCL3, chemokine (C-X-C motif) ligand 8 , and CCL5, resulting in further microglial recruitment. Plaque-associated microglia also release other cytokines, such as IL-6, TNF, macrophage inflammatory peptide- $1 \alpha$, and macrophage colony-stimulating factor [34], which can also serve as chemoattractants $[28,32,33]$.

\section{Receptors Involved in Microglia A $\beta$ Recognition}

Microglia detect fibrillar forms of $A \beta$ though a variety of cell surface pattern recognition receptors, including class A1 scavenger receptors (SCARA1) [35], class B2 scavenger receptors, CD36 [36, 37], and Toll-like receptors (TLRs) 2, 4, and 
6 , and their co-receptor CD14. The role of various microglial scavenger receptors in AD has been thoroughly reviewed in Wilkinson and El Khoury [38]. These receptors act as ensembles to bind fibrillar forms of amyloid and to initiate downstream signaling [38]. In addition, intracellular receptors, such as nucleotide-binding oligomerization domain-like receptors (NLRPs) [39], interact with $\mathrm{A} \beta$, although this has been less well studied.

Stimulation of these microglial receptors by $\mathrm{A} \beta$ results in activation of multiple parallel signal transduction cascades that ultimately stimulate the nuclear factor kappa B (NF-KB)-dependent transcription of proinflammatory cytokines, production of reactive oxygen species, and phagocytosis [40-45]. These responses can be both beneficial and detrimental in the context of AD. Pharmacological upregulation of SCARA1 in monocytes increases their clearance of $A \beta$ [35]. However, SCARA1 also mediates proinflammatory cytokine production, which can have deleterious effects. Similarly, TLRs promote proinflammatory signaling through myeloid differentiation primary response gene 88 or Toll-interleukin receptor-domain-containing adapter-inducing interferon (IFN)- $\beta$-dependent pathways [46]. Deletion of the microglial TLR2/4 co-receptor CD14 reduces the inflammation and amyloid pathology in a transgenic mouse model of AD [47]. Likewise, disruption of TLR signaling by knockout of a common downstream signaling element, IL receptorassociated kinase 4 , leads to decreased levels of brain $\mathrm{A} \beta$ and attenuated microglial reactivity [48]. However, others have shown that mice lacking functional TLR4 have increased microglial activation and brain $A \beta$ levels [49]. Another pathway for proinflammatory microglial activation is the $A \beta$ dependent activation of the inflammasome through NLRP. Microglia in APP/presenilin 1 (PS1) mice deficient in NLRP or caspase-1 are more phagocytic [40]. Genetic deletion of CD36 or antibodies directed to CD36 have been shown to dampen the $A \beta$-induced production of proinflammatory mediators $[36,41]$, although CD36 has been shown to mediate microglial $\mathrm{A} \beta$ phagocytosis and an increase in $\mathrm{CD} 36$ has been linked to clearance of $\mathrm{A} \beta$ from $\mathrm{AD}$ mouse brain $[50,51]$. Together, these $A \beta$ recognition receptors can have dual roles in the disease process, promoting phagocytosis on the one hand, but enhancing inflammation on the other. Because of these dual roles, it is not surprising that the effect of modulating the expression or activation of these receptors has a complex effect on overall amyloid pathology.

\section{Regulation of Microglial Phenotype by Nuclear Receptors}

Nuclear receptors are a class of ligand-activated transcription factors that act to regulate a wide array of cellular responses. Type I nuclear receptors include estrogen and progesterone receptors. The predominant type II receptors are peroxisome proliferator-activated receptors (PPARs) $\gamma, \delta$, and $\alpha$, and liver $\mathrm{X}$ receptors (LXR) $\alpha$ and $\beta$. PPARs function as lipid sensors and are involved in fatty acid metabolism by binding to dietary lipids and their metabolites, while LXRs act as cholesterol sensors. The type II nuclear receptors form obligate heterodimers with retinoid $\mathrm{X}$ receptor to form a functional transcription factor.

In addition to their canonical metabolic roles, PPARs have also been shown to act as "master regulators" of microglial activation. PPAR $\gamma$ expression is required for conversion of macrophages into an anti-inflammatory, prophagocytic phenotype. Arginase 1, a regulator of arginine metabolism which is also commonly associated with an anti-inflammatory microglial phenotype, is a direct target for $\operatorname{PPAR} \gamma[52,53]$. In turn, the prototypic anti-inflammatory cytokine IL-4 induces PPAR $\gamma$ expression through a signal transducer and activator of transcription 6 signaling pathway [54]. In the context of AD, the activation of PPAR $\gamma$ has been shown to promote clearance of $\mathrm{A} \beta$ by "licensing" microglia as competent phagocytes, in part by upregulating CD36 [54]. Consequently, activation of PPAR $\gamma$ leads to rapid reductions in the levels of $\mathrm{A} \beta$ in mouse models of $\mathrm{AD}[51,55-57]$.

Activation of PPAR $\delta$ has similar influences on microglial reactivity. When macrophages engulf apoptotic cells, the catabolism of membrane lipids activates PPAR $\delta$ [58]. Similar to PPAR $\gamma$ [52], genetic inactivation of PPAR $\delta$ [58] and LXRs [59] shift macrophage phenotype towards a proinflammatory state and block their ability to convert into an anti-inflammatory, prophagocytic phenotype. This occurs, in part, through PPAR-mediated transactivation of genes with anti-inflammatory properties, such as IL-10 and TGF- $\beta$. PPARs and LXRs can also promote repression of proinflammatory genes. A subpopulation of PPARs and LXRs can be sumoylated, which enables their interaction with $\mathrm{NF}-\mathrm{KB}$ and activator protein 1 (AP1). This interaction leads to stabilization of the nuclear receptor corepressor 1/histone deacetylase 3 complex with NF- $\mathrm{kB}$ preventing the transcription of $\mathrm{NF}-\kappa \mathrm{B}-$ regulated proinflammatory genes [60]. As a result, genetic deletion of PPAR $\delta$ leads to reduced expression of anti-inflammatory cytokines and impaired phagocytosis. Conversely, activation of PPAR $\delta$ leads to a rapid reduction in brain $A \beta$ deposits in a transgenic mouse model of $\mathrm{AD}[61]$.

\section{Microglial Phagocytosis and Other Endocytic Mechanisms}

Microglia use distinct mechanisms to clear different $A \beta$ species, including phagocytosis, autophagy, and pinocytosis. Impairment of these pathways can result in reduced $A \beta$ clearance in $\mathrm{AD}[62,63]$ (Fig. 1). 


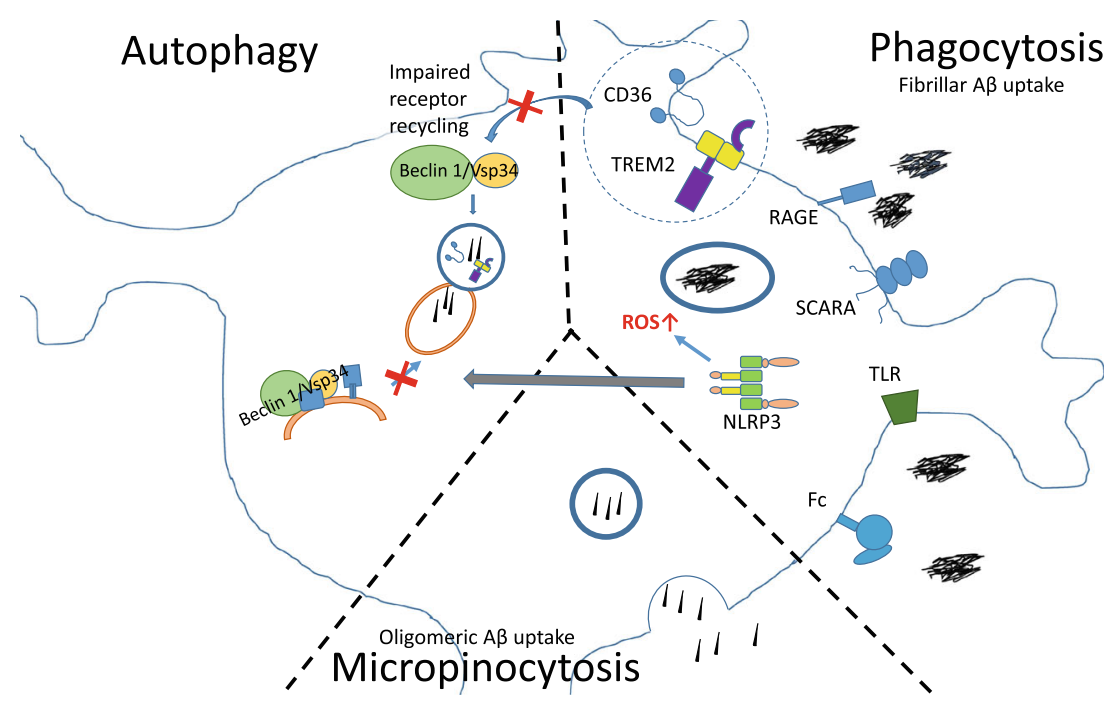

Fig. 1 Multiple pathways for microglial uptake of $\beta$-amyloid $(A \beta)$ are impaired in Alzheimer's disease (AD). Microglia clear $A \beta$ by phagocytosis, micropinocytosis, and autophagy. The figure indicates different receptors capable of recognizing $\mathrm{A} \beta$. In $\mathrm{AD}$ the level of these receptors is downregulated, leading to reduced $\mathrm{A} \beta$ uptake. Reduced levels of beclin 1 lead to impairment in autophagic processes. As beclin 1 also regulates the receptor recycling of CD36 and Trem2, and may promote phagocytic clearance, reduced levels of beclin 1 may also cause defects in phagocytosis. However, inflammasome activation leads to increased formation of reactive oxygen species (ROS), and may also impair autophagy through interaction with beclin 1 . NLRP3 = nucleotide-binding oligomerization domain-like receptor 3; SCARA = class A1 scavenger receptor, TLR = Toll-like receptor; RAGE $=$ receptor for advanced glycation endproducts; $\mathrm{Fc}=\mathrm{Fc}$ receptor

\section{Phagocytosis}

Phagocytosis is one of the key mechanisms for microgliamediated fibrillar and oligomeric $\mathrm{A} \beta$ removal $[22,41,50$, 64-67]. Large particles like $\mathrm{fA} \beta$ are taken up by phagocytosis through engagement of innate immune surface receptors and activation of the cell's phagocytic machinery. After internalization, the phagosomes containing $\mathrm{A} \beta$ are trafficked to lysosomal compartments and degraded by proteases such as cathepsin B [66]. It is of particular interest that microglial phagocytosis is reliant upon beclin 1 , a protein whose actions were thought to be restricted to autophagy. The beclin 1/ Vsp34 complex has been shown to be required for the clearance of apoptotic bodies by macrophages and fibroblasts, and beclin 1-deficient cells are unable to do so [68]. Beclin 1 might promote phagocytic clearance by regulating CD36 and Trem2 expression, as beclin 1 deficiency impairs recycling of these receptors [69]. Lucin et al. [69] have recently reported that beclin 1 levels are reduced in microglia in the AD brain, consistent with the inability of these cells to phagocytose efficiently amyloid deposits.

\section{Autophagy}

Autophagy is an ancient process important for the catabolism of dysfunctional organelles and response to starvation [70]. The principal form of autophagy relevant to neurodegenerative diseases is macroautophagy [71], a process in which molecules are isolated in bulk by autophagosomes. One of the most important mediators of autophagy is the beclin 1/Vsp34 complex. Beclin 1 is an adaptor molecule that stimulates autophagy through promoting vesicle nucleation [72]. Autophagy can be induced by TLR4-mediated activation of myeloid differentiation primary response gene 88 and Toll-interleukin receptordomain-containing adapter-inducing IFN- $\beta$ [73, 74]. These proteins activate TNF receptor associated factor 6 , which induces ubiquitination of beclin 1 , resulting in stimulation of autophagy. However, inflammasome-associated receptors NLRP3, NLRC4, and NLRP4 bind to beclin 1 and reduce autophagy [75]. Several lines of evidence indicate that autophagic processes are impaired in $\mathrm{AD}$, including the fact that beclin 1 levels are reduced in AD brains. Overexpression of beclin 1 reduces APP levels and leads to reduced intracellular and extracellular $\mathrm{A} \beta$ accumulation in $\mathrm{AD}$ mice $[63,76]$. These findings link receptor mediated phagocytosis and autophagy, both of which are impaired in AD.

\section{Macropinocytosis}

Pinocytosis is a mechanism for removal of soluble molecules from the extracellular space, and can occur via 2 pathways, micropinocytosis and macropinocytosis. Micropinocytic vesicles are $<0.1 \mu \mathrm{m}$ in diameter and are typically associated with caveolin. Micropinocytosis does not require actin and occurs within cholesterol-rich lipid domains of the plasma membrane [77]. In contrast, macropinocytosis occurs when membrane ruffles are formed and closed in a process requiring both actin and 
tubulin [78]. In vitro studies have shown that microglial uptake of soluble $A \beta$ is not dependent on the cell surface receptors mediating the uptake of $\mathrm{fA} \beta$ such as scavenger receptors $\mathrm{A}$ and $\mathrm{B}, \mathrm{CD} 36$, and $\mathrm{CD} 47$, and nor does it require the endocytic receptor low-density lipoprotein receptor-related protein-1 [79]. Instead, soluble $A \beta$ is internalized through nonsaturable, fluid-phase macropinocytosis requiring microtubules and actin, and is degraded through the endolytic pathway. Thus, macropinocytosis is a mechanism selective for uptake of soluble and oligomeric $\mathrm{A} \beta$.

\section{The Effect of Microglial Depletion on Plaques}

While subtle alterations in microglial phenotype can have an impact on amyloid pathology, recent studies have used techniques to deplete microglia from the brain to determine the impact of total loss of microglia on AD pathology. Grathwohl et al. [80] demonstrated depleted microglia from APP transgenic mice expressing thymidine kinase of herpex simplex virus under the CD11b promoter (CD11b-HSVTK). In this model microglia could be selectively eliminated on administration of the drug gangciclovir. Surprisingly, microglial depletion had very little effect on plaque formation, plaque maintenance, or neuritic dystrophy [80]. Interestingly, depletion of microglia from the brains of CD11b-HSVTK transgenic mice created a niche for the subsequent infiltration of monocytic cells from the bloodstream [81]. There are caveats to using this genetic model, including myelotoxicity and offtarget effects of ganciclovir on microglial phenotype [80, 82]. Work by Parkhurst et al. [83] took a different approach to depletion of microglia from the brain using a microglialtargeted diphtheria toxin receptor. This study showed that depletion of microglia induced multiple deficits in learning and behavior, and caused a significant reduction in motor learning-dependent synapse formation [83]. This effect was largely due to lack of microglial brain-derived neurotrophic factor signaling as the authors demonstrated that deletion of brain-derived neurotrophic factor specifically in microglia abrogated similar reductions in the levels of GluN2B and VGluT1, as well as impairments in fear conditioning [83]. An important new study has recently demonstrated that microglia can be eliminated from the brain using an orally delivered colony-stimulating factor 1 receptor antagonist [84]. This method for eliminating the microglia did not affect the cognitive functions of the mice. The ability to eliminate these cells easily will allow analysis of the roles of microglia in a number of experimental disease models. The effect of microglial depletion on AD-related behavioral impairments and the role of their blood-derived counterparts remains to be determined.

\section{Effect of Microglial Activation on Neuronal Function in $\mathrm{AD}$}

The effect of microglia on neurons in the context of $\mathrm{AD}$ pathology has been described as detrimental owing to the microglial release of cytotoxic molecules. Elimination of microglia from mixed cultures makes neurons more resistant to the toxic insults elicited by $\mathrm{A} \beta$ in vitro [85]. The toxic role of microglia activation on neurons is supported by studies using $\mathrm{AD}$ mouse models that exhibit direct neuronal loss. In one such study using a model of P301S tauopathy, Yoshiyama et al. [86] reported that microglial activation and synapse loss occur before the appearance of tangles and neuron loss. One mechanism underlying the detrimental effect of microglial activation is the induction of cell cycle events in neurons via TNF- $\alpha$. These cell cycle events are toxic to terminally differentiated neurons [87].

However, the study showing that depletion of microglia does not alter neuritic dystrophy makes it difficult to draw definite conclusions [80]. Indeed, microglial activation may be causing neuronal death and microglia may get activated in response to compromised neuronal function. Most likely, both phenomena exist at the same time, and the vicious inflammatory cycle created by the $\mathrm{AD}$ environment contributes to neuronal loss. Nevertheless, proper communication between neurons and microglia, which is essential for normal brain function, is disrupted in $\mathrm{AD}$.

\section{Regulation of Microglial Phenotype by Neurons}

The interaction between microglia and other cell types is essential for their phenotypic regulation. Neurons regulate microglial activation through interaction of chemokine (CX3-C motif) ligand 1 (CX3CL1) with its receptor CX3CR1, and CD200 with CD200R. Carbohydrates on the neuronal glycocalyx also interact with various siglec receptors on microglia, including the identified AD-associated risk factor CD33. While it does not yet have a definitive ligand, the AD-associated receptor Trem 2 also likely regulates microglial function through contact with cell surface components on other CNS cell types. Together, these interactions are crucially important determinants of microglial phenotype. Not surprisingly, modifying this cell-cell communication has important implications for $\mathrm{AD}$ pathology.

\section{CX3CR1/CX3CL1}

CX3CR1 is a chemokine receptor, expressed exclusively on myeloid cells, that interacts with CX3CL1 (also known as fracktalkine), expressed primarily by neurons $[88,89]$. The expression pattern led researchers to posit that the interaction of CX3CL1/R1 played an important role in communication 
between neurons and microglia [90]. CX3CL1 exists in a membrane-bound form, which acts to promote cell adhesion [91], and a soluble form, which mediates microglial chemoattraction [92]. Hatori et al. [93] also demonstrated that exogenenously applied CX3CL1 could increase microglia proliferation, suggesting that $\mathrm{CX} 3 \mathrm{CL} 1 / \mathrm{R} 1$ signaling could also play a role in neuronal control of microglial abundance. However, perhaps the most dramatic effect of CX3CL1/R1 signaling is the inhibition of microglial inflammatory responses. Cardona et al. [94] showed that deficiency in CX3CR1 caused increased microglial activation and neurotoxicity in multiple neuroinflammatory contexts.

Because CX3CL1/R1 signaling was demonstrated to modify potently microglial phenotypes, Lee et al. [95] examined the effect of knocking out CX3CR1 in APP/PS1 and R1.40 models of AD. As expected from previous findings, CX3CR1 deficiency increased levels of the proinflammatory cytokines TNF- $\alpha$, IL-1 $\beta$ and monocyte chemotactic protein 1 (also known as CCL2). Unexpectedly, however, this resulted in reduced amyloid pathology. These findings were confirmed in additional $\mathrm{AD}$ mouse models [96, 97]. Using in vivo 2photon imaging, Fuhrmann et al. [98] showed that in the 5XFAD model of AD, homozygous loss of CX3CR1 impaired microglia recruitment to areas of neuronal death. This resulted in reduced neurodegeneration, again improving pathology. However, in the hTau model of AD, while CX3CR1 deficiency still enhanced inflammation, it also promoted increased levels of phosphorylated and insoluble tau species [99]. CX3CR1-deficient AD mice also had impaired performance in spatial learning tasks [96, 99]. Thus, CX3CR1/L1 signaling shifts microglia away from a pro-inflammatory phenotype, but this has disparate effects on different aspects of $\mathrm{AD}$ pathology - reducing amyloid pathology on the one hand, while enhancing tau pathology on the other.

\section{CD200/CD200R}

The interaction of CD200 and CD200R is also an important mediator of neuronal regulation of microglial function. In the CNS, CD200 is expressed primarily by neurons, but can, in some inflammatory contexts, also be unregulated on reactive astrocytes [100]. This ligand interacts with the myeloid-cell specific CD200R. Hatherley et al. [101] used targeted mutations to examine the nature of CD200/200R interactions, and showed that the interaction of the ligand receptor pair results in a distance between cells similar to that observed in the immunological synapse. This suggests that CD200/200R binding could facilitate other contact-dependent signaling. Whether by this mechanism or direct signaling through the immunoreceptor tyrosine-based inhibitory motifs on the intracellular domain of CD200R, CD200/200R signaling largely results in downregulation of microglial activation. In CD200deficient mice, microglia express uncharacteristically high levels of the activation markers CD11b and CD45 [102]. The effect of CD200/200R signaling has been well studied in experimental autoimmune encephalomyelitis (EAE) where blocking the CD200 receptor using a monoclonal antibody increases CNS levels of the proinflammatory cytokines IL-6 and IFN- $\gamma$ [103], and inducible nitric oxide synthase [102], resulting in worse clinical scores [104]. Conversely, overexpression of CD200 is protective against EAE induction [105].

Following publication of these findings, researchers began to explore a possible role for CD200/200R signaling in AD. In vitro, the presence of neurons in a microglial culture reduces their inflammatory response to A $\beta$. However, when CD200/ 200R signaling is blocked with a CD200 antibody, the presence of neurons no longer attenuates $A \beta$-induced microglial activation [106]. These findings suggest that, as in EAE, CD200/200R signaling might be neuroprotective in AD. However, CD200 expression is downregulated in rats following intraventricular injection of $A \beta$ [106], and both CD200 and CD200R levels are reduced in patients with $A D$, particularly in parts of the brain that exhibit the highest plaque burden [107]. Thus, downregulation of CD200/200R signaling may provide a permissive environment for development of $\mathrm{AD}$ pathology. Possible mechanisms for $A \beta$-induced downregulation of CD200 and CD200R have recently been explored. Dentesano et al. [108] found that following the administration of lipopolysaccharide (LPS), CEBPB CCAAT/enhancer binding protein- $\beta$ and histone deacetylase 1 bind to the CD200R promoter, resulting in reduced $\mathrm{CD} 200 \mathrm{R}$ expression. They later demonstrated that PPAR $\gamma$ also binds upstream of the CD200R and CD200 genes [109], and that activation of PPAR $\gamma$ prevents the downregulation of $\mathrm{CD} 200 \mathrm{R}$ following exposure to inflammatory mediators. In culture, this translated to enhanced neuroprotection in mixed microglial/neuronal cultures following LPS treatment. While these findings need to be confirmed in the context of $\mathrm{AD}$, these findings suggest potential therapeutic strategies for preventing or reversing the $\mathrm{AD}$ related reduction in CD200/200R signaling.

\section{Siglecs/Sialic Acids}

Siglecs (sialic acid binding immunoglobulin-like lectins) are a subset of lectins that specifically recognize carbohydrates modified with sialic acid residues. The CNS contains high levels of sialic acids, which densely populate the neuronal glycocalyx [110]. During homeostatic conditions, sialic acids on the neuronal cell surface signal that the neuron is healthy and consequently inhibit microglial activation [111].

In addition to their role in homeostasis, siglecs also have been shown to play an important role in neurodegenerative diseases. While there are numerous siglecs involved in the phenotypic regulation of myeloid cells specifically in $\mathrm{AD}$ $[112,113]$, the siglec CD33 has been the best characterized. Upon binding its sialic acid ligand [114], CD33 was shown to 
promote downregulation of myeloid cell activation in the periphery [115]. CD33 function in the CNS, however, was not well studied until a series of genome-wide association studies identified CD33 variants that modified risk for developing $\mathrm{AD}$ [116-118]. It was later shown that CD33 expression levels correlate with cognitive decline in AD patients [119], and that the protective $\mathrm{CD} 33$ variant reduces $\mathrm{CD} 33$ expression [120]. Taken together, these data suggest that CD33 promotes $\mathrm{AD}$ pathogenesis. Consistent with this interpretation, Griciuc et al. [120] showed that upregulation of CD33 in vitro results in reduced $\mathrm{A} \beta$ phagocytosis, while microglial cultured from $\mathrm{CD} 33^{-1-}$ mice exhibit enhanced $\mathrm{A} \beta$ uptake. This was reflected in reduced amyloid pathology in CD33-deficient APP/PS1 mice. Bradshaw et al. [121] reported similar results in monocytes cultured from patients with $\mathrm{AD}$ with a variant of CD33 that increases risk for developing the disease. Monocytes from patients with the $\mathrm{CD} 33$ variant had reduced capacity for $\mathrm{A} \beta$ phagocytosis, and these patients had increased amyloid accumulation in positron emission tomography imaging studies compared with control patients. There are still many aspects of CD33 biology that remain to be explored, including determining a more thorough phenotypic profile of microglia and monocytes expressing the different CD33 variants. Additionally, it will be important to assess the effect of CD33 variants in the context of tau pathology. As studies in CX3CR1deficient mice illustrated, modulation of microglial phenotype can have divergent impact on these 2 pathological hallmarks.

\section{Trem2/Trem2 Ligand}

Trem2 is a single-pass membrane protein expressed in the brain exclusively by microglia, but is also found on peripheral myeloid cells. Studies have yet to identify definitively the ligand of Trem 2 and the cell type on which it is expressed. Stefano et al. [122] used a Trem2-Fc construct to identify heat shock protein 60 (Hsp60) as potential ligand present on neuroblastoma cells. While Hsp60 is normally located in the mitochondria, it can be expressed on the cell surface during cell stress [123], potentially making it accessible to Trem2 in the context of neurodegeneration. In this study, however, it took a very high concentration of Hsp60 to activate Trem2, even on N9 cells, which express unusually high levels of the Trem2 receptor. So, whether a functional interaction occurs between Trem 2 and Hsp60 in vivo remains to be determined. Other groups have identified additional potential Trem2 ligands, including anionic residues on bacteria and lipid molecules [124, 125], as well as undefined species that are abundantly expressed on apoptotic neurons.

While the identity of its physiological ligand is not yet clear, studies have used Trem 2 cross-linking antibodies or Trem2 fusion constructs to study downstream Trem2 signaling pathways and functions. Trem2 requires the intracellular signaling adaptor DNAX activation protein of $12 \mathrm{kDa}$
(DAP12) [126], which, when phosphorylated by the tyrosine kinase src, provides binding sites for the SH2 domaincontaining proteins Syk, phosphoinositide 3-kinase, and DAP10 (reviewed in [127]). Alternatively, SH2-containing inositol-5'-phosphatase 1 can bind to these same sites on DAP12 and prevent downstream signaling through these other pathways [128]. What causes DAP12 to interact with activating or inhibitory components is not yet known. It has been posited to be ligand-specific or to result from the integration of intracellular signals from other activated immune regulatory pathways, though this remains to be further explored.

Trem 2 signaling through these pathways results in regulation of a diverse array of myeloid cell functions. Phagocytosis is the best characterized downstream function of Trem 2 receptor activation. Melchior et al. [129] showed that the level of Trem2 expression in cells of the BV2 rat microglial cell line correlated with their phagocytic capacity. Conversely, knocking down Trem2 in primary microglia inhibits phagocytosis of apoptotic neurons [126]. N'Diaye et al. [130] transfected a Trem2-DAP12 fusion construct into normally nonphagocytic Chinese hamster ovary cells and found that this was sufficient to induce phagocytic uptake of Escherichia coli bioparticles. Trem 2 activation has also been shown to promote anti-inflammatory cytokine production, reduce proinflammatory cytokine production [131], and enhance chemotaxis via upregulation of CCR7 in various immune cell types [132]. While these in vitro studies have determined some of the signaling components involved and possible functional outcomes of Trem 2 signaling, these results need to be confirmed and expanded in an in vivo context.

A role for Trem 2 in AD was first suggested by data that showed that Trem2 was upregulated in plaque-associated myeloid cells in AD mouse models [129, 133]. However, the recent identification of Trem 2 variants that confer a high risk for developing $\mathrm{AD}$ in the human population has greatly accelerated research in this area $[134,135]$. The $\mathrm{R} 47 \mathrm{H}$ variant of Trem 2 confers a 3 -fold increase in risk for $\operatorname{AD}[132,133]$, comparable to the risk conferred by the apolipoprotein E4 allele. Trem 2 mutations are responsible for Nasu-Hakola disease [136]. These findings suggest that Trem 2 may play a fundamental role in disease etiology. Since these studies were published, additional groups have identified Trem 2 variants as genetic risk factors for frontotemporal dementia [137], Parkinson's disease [138], and amyotrophic lateral sclerosis [139]. This common genetic linkage suggests a more general role for Trem 2 in modulating neurodegenerative disease pathology.

The human genetic data have instigated a number of studies to examine possible mechanisms by which these Trem2 variants confer increased risk for AD. Because a missense mutation is among the Trem2 variants identified as increasing $\mathrm{AD}$ risk, it has been posited that these Trem 2 variants cause loss of Trem2 function [140]. While this still needs to be 
explored experimentally, the studies published thus far focus on Trem 2 deficiency in AD models. Jiang et al. [141] induced overexpression of Trem 2 by intraventicular lentrivral injection in APPswe/PS1de9 mice. As expected, overexpressing Trem2 resulted in reduced levels of the proinflammatory cytokines TNF- $\alpha$, IL-1 $\beta$, IL- 6 , and inducible nitric oxide synthase; reduced amyloid pathology; and improved behavior. However, Ulrich et al. [142] showed trends but no significant changes in inflammatory markers or amyloid pathology in APPPS1;Trem $2^{+/}$mice. Jay et al. showed opposing data, namely that APPPS1;Trem $2^{-1-}$ mice exhibit reduced levels of proinflammatory cytokines, increased levels of antiinflammatory cytokines, and ameliorated amyloid and tau pathology (unpublished data). These studies might have divergent results owing to alterations in the role of Trem2 throughout the progression of $\mathrm{AD}$ pathology, differences in acute versus chronic changes in Trem2 levels, or because of differences in the specific cell types targeted in these various studies. Others have examined changes in Trem2-deficient mice in the context of stroke and shown increased inflammation in Trem $2^{-/-}$mice [143], whereas blocking Trem2 using a monoclonal antibody in the context of EAE blocked infiltration of peripheral immune cells and ameliorated pathology [144]. This provides additional evidence that the role of Trem 2 might be context dependent. As these issues continue to be explored, the biology of Trem 2 may have to be redefined to fit with these new in vivo findings.

\section{Infiltration of Peripheral Monocytes into the AD Brain}

It is generally accepted that microglia can become dysfunctional during aging and disease $[145,146]$. In order to compensate for disease-related dysfunction or to respond to injury or pathogens, microglial density can increase dramatically in these contexts [33]. However, it is controversial as to how changes in microglial number in these settings is achieved. A number of studies suggested that peripheral monocytes might enter the CNS and differentiate into microglia, but lethal whole-body irradiation induced confounds that called these results into question [147, 148].

While the origin of microglia, particularly the expanded population evident in disease, has historically always been controversial, a series of studies in the mid-2000s suggested that brain macrophages derived from peripheral monocytes were important players in AD. Malm et al. [149] used irradiation and transplantation of bone marrow from an enhanced green fluorescent protein donor to label specifically peripheral hematopoietic cells in APP/PS1 mice. They found that some of these cells localized around $A \beta$ deposits. This association was increased following LPS injection and resulted in reduced A $\beta$ burden. Simard et al. [150] used the same technique and also found that peripherally derived cells were recruited to the brain in response to $A \beta 40$ and $A \beta 42$ species and, once extravasated, migrated to $A \beta$ plaques. By depleting microglia using the HSVTK strategy discussed above, and allowing several months for repopulation to occur, they showed that these mice had improved amyloid pathology. The plaqueassociated myeloid cells had reduced levels of the proinflammatory genes $I L 1 \beta$ and $T L R 2$, and actively phagocytosed $\mathrm{A} \beta$, suggesting that these presumably peripherally derived cells were beneficial in this disease model. El Khoury et al. [33] showed that the trafficking of peripherally derived cells was greatly reduced in $\mathrm{CCR} 2^{--}$mice. Furthermore, these mice had enhanced amyloid accumulation. These findings suggests that peripheral immune cells are responsible for the increase in myeloid cell number in $\mathrm{AD}$, and play an important role in $\mathrm{A} \beta$ clearance. The importance of CCR2 + cells in $\mathrm{AD}$ was later confirmed in studies which showed that knocking out CCR2 in APP/PS1 mice reduced inflammatory markers in cells surrounding plaques, while enhancing soluble $A \beta$ levels and resulting in worse performance on spatial memory tests [151]. Taken together, these results from chimeras and evidence from CCR2-deficient mice suggested that peripheral monocytes infiltrated into the $\mathrm{AD}$ brain, migrated to $\mathrm{A} \beta$ plaques, and modulated AD pathology and behavior.

However, the outcome of studies using bone marrow chimeras were later called into question. These studies relied on irradiation and bone marrow transplantation as a primary means to distinguish peripherally derived cells from resident microglia. Mildner et al. [147] showed that parenchymal microglia can arise from CCR2+Ly6Chi monocytes, but that this did not occur without preconditioning irradiation of chimeras. They showed that whole body irradiation was necessary to induce trafficking of peripheral cells into the CNS. These effects were long lasting, with increases in peripheral immune cell trafficking into the CNS several months after irradiation. Additionally, Ajami et al. [148] showed that bone marrow transplantation could cause aberrant cell infiltration into the CNS, even in the absence of irradiation. They posited that the presence of hematopoietic precursors in the bloodstream following bone marrow transplantation could artificially increase trafficking of peripherally derived cells into the CNS.

The controversy regarding peripheral cell infiltration in $\mathrm{AD}$ was echoed in studies looking at repopulation of microglia following depletion. Varvel et al. [81] depleted microglia by expressing HSVTK under the control of the CD11b promoter. They used bone marrow transplantation from a congenic wildtype mouse to prevent depletion of peripheral immune cells. They found that 2 weeks after microglial depletion, the brain was completely repopulated $(2 \times$ tiling density) by CCR $2+$ peripheral monocytes. However, a recent study using a different depletion model showed that repopulating cells were derived from resident microglial progenitors rather than from 
peripheral infiltrates [84]. It is possible that monocyte entry into the CNS is dependent on the particular experimental paradigm, but this controversy remains to be resolved.

Most of these studies suggest that, if peripheral monocytes play a role in $\mathrm{AD}$, the proinflammatory $\mathrm{CCR} 2+$ subset of monocytes is likely important. Characteristically, Ly $6 \mathrm{C}^{\mathrm{hi}} \mathrm{CX} 3 \mathrm{CR} 1^{-} \mathrm{CCR} 2^{+}$cells infiltrate tissue during inflammation [152], while the $\mathrm{Ly}_{6 \mathrm{C}^{\mathrm{lo}}} \mathrm{CX} 3 \mathrm{CR} 1+\mathrm{CCR} 2^{-}$monocytes perform homeostatic functions [153]. The Ly6 $\mathrm{C}^{\text {hi }}$ cells downregulate Ly6C over time and change monocyte subtype in the periphery [153]. The role of CCR2 in infiltration of peripheral monocytes has been established in the genetic depletion models discussed above. Additionally, viral-mediated overexpression of its ligand CCL2 in the CNS mediates enhanced peripheral immune cell trafficking [154]. However, it remains to be seen whether the CX3CR1+ monocyte subtype could also play a role in $\mathrm{AD}$ pathogenesis.

A recent study provides novel evidence that microglia derived from peripheral monocytes could play a role in $\mathrm{AD}$. Jay et al. (unpublished data) showed that Trem2+ cells are plaque-associated myeloid cells in human patients with $\mathrm{AD}$ cases and $3 \mathrm{AD}$ mouse models. Using flow cytometry, they showed that these Trem2+ cells are exclusively CD45hi, a classic marker of peripherally derived macrophages in the CNS. While these cells are highly immunoreactive with CD45, they do not label with the marker P2RY12, a purinergic receptor found to be specifically expressed exclusively in microglia but not peripherally derived cell populations [155]. Interestingly, these CD45hi, plaque-associated cells are absent in APPPS1;Trem $2^{-/}$mice. While these data still require confirmation using additional techniques, if the Trem $2+$ cell population is peripherally derived, the high risk conferred by Trem 2 variant mutations suggest a critical role for peripheral monocytes in $\mathrm{AD}$ etiology.

\section{Conclusions}

Microglial dysfunction is largely responsible for the overwhelmed inflammatory milieu in AD brain. Research has pinpointed key molecules with ability to shift microglial phenotype and induce phagocytosis. Successful engagement of these processes is likely to be of great therapeutic benefit.

Required Author Forms Disclosure forms provided by the authors are available with the online version of this article.

\section{References}

1. Lawson LJ, Perry VH, Dri P, Gordon S. Heterogeneity in the distribution and morphology of microglia in the normal adult mouse brain. Neuroscience 1990;39:151-170.
2. Alliot F, Godin I, Pessac B. Microglia derive from progenitors, originating from the yolk sac, and which proliferate in the brain. Brain Res Dev Brain Res 1999;117:145-152.

3. Ginhoux F, Greter M, Leboeuf M, et al. Fate mapping analysis reveals that adult microglia derive from primitive macrophages. Science 2010;330:841-845.

4. Nimmerjahn A, Kirchhoff F, Helmchen F. Resting microglial cells are highly dynamic surveillants of brain parenchyma in vivo. Science 2005;308:1314-1318.

5. Davalos D, Grutzendler J, Yang G, et al. ATP mediates rapid microglial response to local brain injury in vivo. Nat Neurosci 2005;8:752-758.

6. Ransohoff RM, Stevens B. Neuroscience. How many cell types does it take to wire a brain? Science 2011;333:1391-1392.

7. Haynes SE, Hollopeter G, Yang G, et al. The P2Y12 receptor regulates microglial activation by extracellular nucleotides. Nat Neurosci 2006;9:1512-1519.

8. Wake H, Moorhouse AJ, Jinno S, Kohsaka S, Nabekura J. Resting microglia directly monitor the functional state of synapses in vivo and determine the fate of ischemic terminals. J Neurosci 2009;29: 3974-3980.

9. McGeer PL, Rogers J. Anti-inflammatory agents as a therapeutic approach to Alzheimer's disease. Neurology 1992;42:447-449.

10. Akiyama H, Mori H, Saido T, Kondo H, Ikeda K, McGeer PL. Occurrence of the diffuse amyloid beta-protein (Abeta) deposits with numerous Abeta-containing glial cells in the cerebral cortex of patients with alzheimer's disease. Glia 1999;25:324-331.

11. Mackenzie IR, Hao C, Munoz DG. Role of microglia in senile plaque formation. Neurobiol Aging 1995;16:797-804.

12. Ferrera D, Mazzaro N, Canale C, Gasparini L. Resting microglia react to Abeta42 fibrils but do not detect oligomers or oligomerinduced neuronal damage. Neurobiol Aging 2014;35:2444-2457.

13. Hanzel CE, Pichet-Binette A, Pimentel LS, et al. Neuronal driven pre-plaque inflammation in a transgenic rat model of alzheimer's disease. Neurobiol Aging 2014;35:2249-2262.

14. Meyer-Luehmann M, Spires-Jones TL, Prada C, et al. Rapid appearance and local toxicity of amyloid-beta plaques in a mouse model of Alzheimer's disease. Nature 2008;451:720-724.

15. Bolmont T, Haiss F, Eicke D, et al. Dynamics of the microglial/ amyloid interaction indicate a role in plaque maintenance. $\mathrm{J}$ Neurosci 2008;28:4283-4292.

16. Holmes C, Cunningham C, Zotova E, et al. Systemic inflammation and disease progression in Alzheimer disease. Neurology 2009;73: $768-774$.

17. Liao YF, Wang BJ, Cheng HT, Kuo LH, Wolfe MS. Tumor necrosis factor-alpha, interleukin-1beta, and interferon-gamma stimulate gamma-secretase-mediated cleavage of amyloid precursor protein through a JNK-dependent MAPK pathway. J Biol Chem 2004;279: 49523-49532.

18. Blasko I, Veerhuis R, Stampfer-Kountchev M, Saurwein-Teissl M, Eikelenboom P, Grubeck-Loebenstein B. Costimulatory effects of interferon-gamma and interleukin-1beta or tumor necrosis factor alpha on the synthesis of Abeta1-40 and Abeta1-42 by human astrocytes. Neurobiol Dis 2000;7:682-689.

19. Hong HS, Hwang EM, Sim HJ, et al. Interferon gamma stimulates beta-secretase expression and sAPPbeta production in astrocytes. Biochem Biophys Res Commun 2003;307:922-927.

20. Chung H, Brazil MI, Soe TT, Maxfield FR. Uptake, degradation, and release of fibrillar and soluble forms of alzheimer's amyloid beta-peptide by microglial cells. J Biol Chem 1999;274:3230132308 .

21. Hickman SE, El Khoury J. The neuroimmune system in Alzheimer's disease: The glass is half full. J Alzheimers Dis 2013;33(Suppl. 1):S295-S302.

22. Koenigsknecht-Talboo J, Landreth GE. Microglial phagocytosis induced by fibrillar beta-amyloid and IgGs are differentially 
regulated by proinflammatory cytokines. J Neurosci 2005;25:82408249.

23. Orre M, Kamphuis W, Osborn LM, et al. Isolation of glia from Alzheimer's mice reveals inflammation and dysfunction. Neurobiol Aging 2014.

24. Bard F, Cannon C, Barbour R, et al. Peripherally administered antibodies against amyloid beta-peptide enter the central nervous system and reduce pathology in a mouse model of alzheimer disease. Nat Med 2000;6:916-919.

25. Schenk D, Barbour R, Dunn W, et al. Immunization with amyloidbeta attenuates alzheimer-disease-like pathology in the PDAPP mouse. Nature 1999;400:173-177.

26. Gertig U, Hanisch UK. Microglial diversity by responses and responders. Front Cell Neurosci 2014;8:101.

27. Boddeke EW, Meigel I, Frentzel S, et al. Cultured rat microglia express functional beta-chemokine receptors. J Neuroimmunol 1999;98:176-184.

28. Guillot-Sestier MV, Town T. Innate immunity in Alzheimer's disease: A complex affair. CNS Neurol Disord Drug Targets 2013;12:593-607.

29. Oddo S, Caccamo A, Shepherd JD, et al. Triple-transgenic model of Alzheimer's disease with plaques and tangles: Intracellular abeta and synaptic dysfunction. Neuron 2003;39:409-421.

30. Janelsins MC, Mastrangelo MA, Oddo S, LaFerla FM, Federoff HJ, Bowers WJ. Early correlation of microglial activation with enhanced tumor necrosis factor-alpha and monocyte chemoattractant protein-1 expression specifically within the entorhinal cortex of triple transgenic Alzheimer's disease mice. J Neuroinflammation 2005;2:23.

31. Jankowsky JL, Fadale DJ, Anderson J, et al. Mutant presenilins specifically elevate the levels of the 42 residue beta-amyloid peptide in vivo: Evidence for augmentation of a 42-specific gamma secretase. Hum Mol Genet 2004;13:159-170.

32. Ishizuka K, Kimura T, Igata-yi R, Katsuragi S, Takamatsu J, Miyakawa T. Identification of monocyte chemoattractant protein-1 in senile plaques and reactive microglia of Alzheimer's disease. Psychiatry Clin Neurosci 1997;51:135-138.

33. El Khoury J, Toft M, Hickman SE, et al. Cer2 deficiency impairs microglial accumulation and accelerates progression of Alzheimerlike disease. Nat Med 2007;13:432-438.

34. Naert G, Rivest S. CC chemokine receptor 2 deficiency aggravates cognitive impairments and amyloid pathology in a transgenic mouse model of Alzheimer's disease. J Neurosci 2011;31:62086220.

35. Frenkel D, Wilkinson K, Zhao L, et al. Scaral deficiency impairs clearance of soluble amyloid-beta by mononuclear phagocytes and accelerates Alzheimer's-like disease progression. Nat Commun 2013;4:2030.

36. El Khoury JB, Moore KJ, Means TK, et al. CD36 mediates the innate host response to beta-amyloid. J Exp Med 2003;197:16571666.

37. Coraci IS, Husemann J, Berman JW, et al. CD36, a class B scavenger receptor, is expressed on microglia in Alzheimer's disease brains and can mediate production of reactive oxygen species in response to beta-amyloid fibrils. Am J Pathol 2002;160:101-112.

38. Wilkinson K, El Khoury J. Microglial scavenger receptors and their roles in the pathogenesis of Alzheimer's disease. Int J Alzheimers Dis 2012;2012:489456.

39. Halle A, Hornung V, Petzold GC, et al. The NALP3 inflammasome is involved in the innate immune response to amyloid-beta. Nat Immunol 2008;9:857-865.

40. Heneka MT, Kummer MP, Stutz A, et al. NLRP3 is activated in Alzheimer's disease and contributes to pathology in APP/PS1 mice. Nature 2013;493:674-678.

41. Bamberger ME, Harris ME, McDonald DR, Husemann J, Landreth GE. A cell surface receptor complex for fibrillar beta-amyloid mediates microglial activation. J Neurosci 2003;23:2665-2674.
42. McDonald DR, Bamberger ME, Combs CK, Landreth GE. Betaamyloid fibrils activate parallel mitogen-activated protein kinase pathways in microglia and THP1 monocytes. J Neurosci 1998;18: $4451-4460$.

43. Alarcon R, Fuenzalida C, Santibanez M, von Bernhardi R. Expression of scavenger receptors in glial cells. comparing the adhesion of astrocytes and microglia from neonatal rats to surface-bound beta-amyloid. J Biol Chem 2005;280:30406-30415.

44. Fang F, Lue LF, Yan S, et al. RAGE-dependent signaling in microglia contributes to neuroinflammation, Abeta accumulation, and impaired learning/memory in a mouse model of Alzheimer's disease. FASEB J 2010;24:1043-1055.

45. Chung H, Brazil MI, Irizarry MC, Hyman BT, Maxfield FR. Uptake of fibrillar beta-amyloid by microglia isolated from MSR-A (type I and type II) knockout mice. Neuroreport 2001;12:1151-1154.

46. Landreth GE, Reed-Geaghan EG. Toll-like receptors in Alzheimer's disease. Curr Top Microbiol Immunol 2009;336:137-153.

47. Reed-Geaghan EG, Reed QW, Cramer PE, Landreth GE. Deletion of CD14 attenuates Alzheimer's disease pathology by influencing the brain's inflammatory milieu. J Neurosci 2010;30:15369-15373.

48. Cameron B, Tse W, Lamb R, Li X, Lamb BT, Landreth GE. Loss of interleukin receptor-associated kinase 4 signaling suppresses amyloid pathology and alters microglial phenotype in a mouse model of Alzheimer's disease. J Neurosci 2012;32:15112-15123.

49. Song M, Jin J, Lim JE, et al. TLR4 mutation reduces microglial activation, increases $A \beta$ deposits and exacerbates cognitive deficits in a mouse model of Alzheimer's disease. J Neuroinflammation 2011;8:92-2094-8-92.

50. Koenigsknecht J, Landreth G. Microglial phagocytosis of fibrillar beta-amyloid through a betal integrin-dependent mechanism. J Neurosci 2004;24:9838-9846.

51. Yamanaka M, Ishikawa T, Griep A, Axt D, Kummer MP, Heneka MT. PPARgamma/RXRalpha-induced and CD36-mediated microglial amyloid-beta phagocytosis results in cognitive improvement in amyloid precursor protein/presenilin 1 mice. J Neurosci 2012;32:17321-17331.

52. Odegaard JI, Ricardo-Gonzalez RR, Goforth MH, et al. Macrophage-specific PPARgamma controls alternative activation and improves insulin resistance. Nature 2007;447:1116-1120.

53. Gallardo-Soler A, Gomez-Nieto C, Campo ML, et al. Arginase I induction by modified lipoproteins in macrophages: A peroxisome proliferator-activated receptor-gamma/delta-mediated effect that links lipid metabolism and immunity. Mol Endocrinol 2008;22: 1394-1402.

54. Szanto A, Balint BL, Nagy ZS, et al. STAT6 transcription factor is a facilitator of the nuclear receptor PPARgamma-regulated gene expression in macrophages and dendritic cells. Immunity 2010;33: 699-712.

55. Escribano L, Simon AM, Gimeno E, et al. Rosiglitazone rescues memory impairment in alzheimer's transgenic mice: Mechanisms involving a reduced amyloid and tau pathology. Neuropsychopharmacology 2010;35:1593-1604.

56. Pedersen WA, McMillan PJ, Kulstad JJ, Leverenz JB, Craft S, Haynatzki GR. Rosiglitazone attenuates learning and memory deficits in Tg2576 Alzheimer mice. Exp Neurol 2006;199:265-273.

57. Mandrekar-Colucci S, Karlo JC, Landreth GE. Mechanisms underlying the rapid peroxisome proliferator-activated receptor-gammamediated amyloid clearance and reversal of cognitive deficits in a murine model of Alzheimer's disease. J Neurosci 2012;32:1011710128.

58. Mukundan L, Odegaard JI, Morel CR, et al. PPAR-delta senses and orchestrates clearance of apoptotic cells to promote tolerance. Nat Med 2009;15:1266-1272.

59. A-Gonzalez N, Bensinger SJ, Hong C, et al. Apoptotic cells promote their own clearance and immune tolerance through activation of the nuclear receptor LXR. Immunity 2009;31:245-258. 
60. Glass CK, Saijo K. Nuclear receptor transrepression pathways that regulate inflammation in macrophages and $\mathrm{T}$ cells. Nat Rev Immunol 2010;10:365-376.

61. Kalinin S, Richardson JC, Feinstein DL. A PPARdelta agonist reduces amyloid burden and brain inflammation in a transgenic mouse model of Alzheimer's disease. Curr Alzheimer Res 2009;6: 431-437.

62. Nixon RA, Wegiel J, Kumar A, et al. Extensive involvement of autophagy in alzheimer disease: An immuno-electron microscopy study. J Neuropathol Exp Neurol 2005;64:113-122.

63. Pickford F, Masliah E, Britschgi M, et al. The autophagy-related protein beclin 1 shows reduced expression in early alzheimer disease and regulates amyloid beta accumulation in mice. J Clin Invest 2008;118:2190-2199.

64. Reed-Geaghan EG, Savage JC, Hise AG, Landreth GE. CD14 and toll-like receptors 2 and 4 are required for fibrillar A\{beta\}-stimulated microglial activation. J Neurosci 2009;29:11982-11992.

65. Richard KL, Filali M, Prefontaine P, Rivest S. Toll-like receptor 2 acts as a natural innate immune receptor to clear amyloid beta 1-42 and delay the cognitive decline in a mouse model of Alzheimer's disease. J Neurosci 2008;28:5784-5793.

66. Yang CN, Shiao YJ, Shie FS, et al. Mechanism mediating oligomeric Abeta clearance by naive primary microglia. Neurobiol Dis 2011;42:221-230.

67. Knauer MF, Soreghan B, Burdick D, Kosmoski J, Glabe CG. Intracellular accumulation and resistance to degradation of the alzheimer amyloid A4/beta protein. Proc Natl Acad Sci U S A 1992;89:7437-7441.

68. Konishi A, Arakawa S, Yue Z, Shimizu S. Involvement of beclin 1 in engulfment of apoptotic cells. J Biol Chem 2012;287:1391913929.

69. Lucin KM, O’Brien CE, Bieri G, et al. Microglial beclin 1 regulates retromer trafficking and phagocytosis and is impaired in Alzheimer's disease. Neuron 2013;79:873-886.

70. Deretic V, Levine B. Autophagy, immunity, and microbial adaptations. Cell Host Microbe 2009;5:527-549.

71. Todde V, Veenhuis M, van der Klei IJ. Autophagy: Principles and significance in health and disease. Biochim Biophys Acta 2009;1792:3-13.

72. Suzuki K, Kirisako T, Kamada Y, Mizushima N, Noda T, Ohsumi Y. The pre-autophagosomal structure organized by concerted functions of APG genes is essential for autophagosome formation. EMBO J 2001;20:5971-5981.

73. Shi CS, Kehrl JH. TRAF6 and A20 regulate lysine 63-linked ubiquitination of beclin-1 to control TLR4-induced autophagy. Sci Signal 2010;3:ra42.

74. Shi CS, Kehrl JH. MyD88 and trif target beclin 1 to trigger autophagy in macrophages. J Biol Chem 2008;283:33175-33182.

75. Jounai N, Kobiyama K, Shiina M, Ogata K, Ishii KJ, Takeshita F. NLRP4 negatively regulates autophagic processes through an association with beclin1. J Immunol 2011;186:1646-1655.

76. Jaeger PA, Pickford F, Sun CH, Lucin KM, Masliah E, Wyss-Coray $\mathrm{T}$. Regulation of amyloid precursor protein processing by the beclin 1 complex. PLoS One 2010;5:e11102.

77. Parton RG, Richards AA. Lipid rafts and caveolae as portals for endocytosis: New insights and common mechanisms. Traffic 2003;4:724-738.

78. Conner SD, Schmid SL. Regulated portals of entry into the cell. Nature 2003;422:37-44.

79. Mandrekar S, Jiang Q, Lee CY, Koenigsknecht-Talboo J, Holtzman DM, Landreth GE. Microglia mediate the clearance of soluble abeta through fluid phase macropinocytosis. J Neurosci 2009;29:42524262 .

80. Grathwohl SA, Kalin RE, Bolmont T, et al. Formation and maintenance of alzheimer's disease beta-amyloid plaques in the absence of microglia. Nat Neurosci 2009;12:1361-1363.
81. Varvel NH, Grathwohl SA, Baumann F, et al. Microglial repopulation model reveals a robust homeostatic process for replacing CNS myeloid cells. Proc Natl Acad Sci U S A 2012;109:18150-18155.

82. Heppner FL, Greter M, Marino D, et al. Experimental autoimmune encephalomyelitis repressed by microglial paralysis. Nat Med 2005;11:146-152.

83. Parkhurst CN, Yang G, Ninan I, et al. Microglia promote learningdependent synapse formation through brain-derived neurotrophic factor. Cell 2013;155:1596-1609.

84. Elmore MR, Najafi AR, Koike MA, et al. Colony-stimulating factor 1 receptor signaling is necessary for microglia viability, unmasking a microglia progenitor cell in the adult brain. Neuron 2014;82:380 397.

85. Giulian D, Haverkamp LJ, Yu JH, et al. Specific domains of betaamyloid from alzheimer plaque elicit neuron killing in human microglia. J Neurosci 1996;16:6021-6037.

86. Yoshiyama Y, Higuchi M, Zhang B, et al. Synapse loss and microglial activation precede tangles in a P301S tauopathy mouse model. Neuron 2007;53:337-351.

87. Bhaskar K, Maphis $\mathrm{N}, \mathrm{Xu}$ G, et al. Microglial derived tumor necrosis factor-alpha drives Alzheimer's disease-related neuronal cell cycle events. Neurobiol Dis 2014;62:273-285.

88. Jung S, Aliberti J, Graemmel P, et al. Analysis of fractalkine receptor $\mathrm{CX}(3) \mathrm{CR} 1$ function by targeted deletion and green fluorescent protein reporter gene insertion. Mol Cell Biol. 2000;20: 4106-4114

89. Nishiyori A, Minami M, Ohtani Y, et al. Localization of fractalkine and CX3CR1 mRNAs in rat brain: Does fractalkine play a role in signaling from neuron to microglia? FEBS Lett 1998;429:167-172.

90. Harrison JK, Jiang Y, Chen S, et al. Role for neuronally derived fractalkine in mediating interactions between neurons and CX3CR1-expressing microglia. Proc Natl Acad Sci U S A 1998;95:10896-10901.

91. Haskell CA, Cleary MD, Charo IF. Molecular uncoupling of fractalkine-mediated cell adhesion and signal transduction. rapid flow arrest of CX3CR1-expressing cells is independent of Gprotein activation. J Biol Chem 1999;274:10053-10058.

92. Maciejewski-Lenoir D, Chen S, Feng L, Maki R, Bacon KB. Characterization of fractalkine in rat brain cells: Migratory and activation signals for CX3CR-1-expressing microglia. J Immunol 1999;163:1628-1635.

93. Hatori K, Nagai A, Heisel R, Ryu JK, Kim SU. Fractalkine and fractalkine receptors in human neurons and glial cells. J Neurosci Res 2002;69:418-426.

94. Cardona AE, Pioro EP, Sasse ME, et al. Control of microglial neurotoxicity by the fractalkine receptor. Nat Neurosci 2006;9: 917-924.

95. Lee S, Varvel NH, Konerth ME, et al. CX3CR1 deficiency alters microglial activation and reduces beta-amyloid deposition in two Alzheimer's disease mouse models. Am J Pathol 2010;177:2549-2562.

96. Cho SH, Sun B, Zhou Y, et al. CX3CR1 protein signaling modulates microglial activation and protects against plaque-independent cognitive deficits in a mouse model of alzheimer disease. J Biol Chem 2011;286:32713-32722.

97. Liu Z, Condello C, Schain A, Harb R, Grutzendler J. CX3CR1 in microglia regulates brain amyloid deposition through selective protofibrillar amyloid-beta phagocytosis. J Neurosci 2010;30: 17091-17101.

98. Fuhrmann M, Bittner T, Jung CK, et al. Microglial Cx3cr1 knockout prevents neuron loss in a mouse model of Alzheimer's disease. Nat Neurosci 2010;13:411-413.

99. Bhaskar K, Konerth M, Kokiko-Cochran ON, Cardona A, Ransohoff RM, Lamb BT. Regulation of tau pathology by the microglial fractalkine receptor. Neuron 2010;68:19-31.

100. Koning N, Swaab DF, Hoek RM, Huitinga I. Distribution of the immune inhibitory molecules CD200 and CD200R in the normal 
central nervous system and multiple sclerosis lesions suggests neuron-glia and glia-glia interactions. J Neuropathol Exp Neurol 2009;68:159-167.

101. Hatherley D, Lea SM, Johnson S, Barclay AN. Structures of CD200/CD200 receptor family and implications for topology, regulation, and evolution. Structure 2013;21:820-832.

102. Hoek RM, Ruuls SR, Murphy CA, et al. Down-regulation of the macrophage lineage through interaction with OX2 (CD200). Science 2000;290:1768-1771.

103. Meuth SG, Simon OJ, Grimm A, et al. CNS inflammation and neuronal degeneration is aggravated by impaired CD200CD200R-mediated macrophage silencing. J Neuroimmunol 2008;194:62-69.

104. Wright GJ, Cherwinski H, Foster-Cuevas M, et al. Characterization of the CD200 receptor family in mice and humans and their interactions with CD200. J Immunol 2003;171:3034-3046.

105. Chitnis T, Imitola J, Wang Y, et al. Elevated neuronal expression of CD200 protects wlds mice from inflammation-mediated neurodegeneration. Am J Pathol 2007;170:1695-1712.

106. Lyons A, Downer EJ, Crotty S, Nolan YM, Mills KH, Lynch MA. CD200 ligand receptor interaction modulates microglial activation in vivo and in vitro: A role for IL-4. J Neurosci 2007;27:8309-8313.

107. Walker DG, Dalsing-Hernandez JE, Campbell NA, Lue LF. Decreased expression of CD200 and CD200 receptor in Alzheimer's disease: A potential mechanism leading to chronic inflammation. Exp Neurol 2009;215:5-19.

108. Dentesano G, Straccia M, Ejarque-Ortiz A, et al. Inhibition of CD200R1 expression by C/EBP beta in reactive microglial cells. J Neuroinflammation 2012;9:165-2094-9-165.

109. Dentesano G, Serratosa J, Tusell JM, et al. CD200R1 and CD200 expression are regulated by PPAR-gamma in activated glial cells. Glia 2014;62:982-998.

110. Crocker PR, Paulson JC, Varki A. Siglecs and their roles in the immune system. Nat Rev Immunol 2007;7:255-266.

111. Linnartz B, Neumann H. Microglial activatory (immunoreceptor tyrosine-based activation motif)- and inhibitory (immunoreceptor tyrosine-based inhibition motif)-signaling receptors for recognition of the neuronal glycocalyx. Glia 2013;61:37-46.

112. Wang Y, Neumann H. Alleviation of neurotoxicity by microglial human siglec-11. J Neurosci 2010;30:3482-3488.

113. Lunnon K, Teeling JL, Tutt AL, Cragg MS, Glennie MJ, Perry VH. Systemic inflammation modulates fc receptor expression on microglia during chronic neurodegeneration. J Immunol 2011;186:72157224.

114. Lajaunias F, Dayer JM, Chizzolini C. Constitutive repressor activity of CD33 on human monocytes requires sialic acid recognition and phosphoinositide 3-kinase-mediated intracellular signaling. Eur J Immunol 2005;35:243-251.

115. Paul SP, Taylor LS, Stansbury EK, McVicar DW. Myeloid specific human CD33 is an inhibitory receptor with differential ITIM function in recruiting the phosphatases SHP-1 and SHP-2. Blood 2000;96:483-490.

116. Bertram L, Lange C, Mullin K, et al. Genome-wide association analysis reveals putative alzheimer's disease susceptibility loci in addition to APOE. Am J Hum Genet 2008;83:623-632.

117. Naj AC, Jun G, Beecham GW, et al. Common variants at MS4A4/ MS4A6E, CD2AP, CD33 and EPHA1 are associated with late-onset Alzheimer's disease. Nat Genet 2011;43:436-441.

118. Hollingworth P, Harold D, Sims R, et al. Common variants at ABCA7, MS4A6A/MS4A4E, EPHA1, CD33 and CD2AP are associated with Alzheimer's disease. Nat Genet 2011;43:429435

119. Karch CM, Jeng AT, Nowotny P, Cady J, Cruchaga C, Goate AM. Expression of novel Alzheimer's disease risk genes in control and Alzheimer's disease brains. PLoS One 2012;7: e50976.
120. Griciuc A, Serrano-Pozo A, Parrado AR, et al. Alzheimer's disease risk gene CD33 inhibits microglial uptake of amyloid beta. Neuron 2013;78:631-643.

121. Bradshaw EM, Chibnik LB, Keenan BT, et al. CD33 Alzheimer's disease locus: Altered monocyte function and amyloid biology. Nat Neurosci 2013;16:848-850.

122. Stefano L, Racchetti G, Bianco F, et al. The surface-exposed chaperone, Hsp60, is an agonist of the microglial TREM2 receptor. J Neurochem 2009;110:284-294.

123. Goh YC, Yap CT, Huang BH, et al. Heat-shock protein 60 translocates to the surface of apoptotic cells and differentiated megakaryocytes and stimulates phagocytosis. Cell Mol Life Sci 2011;68:15811592.

124. Daws MR, Sullam PM, Niemi EC, Chen TT, Tchao NK, Seaman WE. Pattern recognition by TREM-2: Binding of anionic ligands. J Immunol 2003;171:594-599.

125. Cannon JP, O'Driscoll M, Litman GW. Specific lipid recognition is a general feature of CD300 and TREM molecules. Immunogenetics 2012;64:39-47.

126. Takahashi K, Rochford CD, Neumann H. Clearance of apoptotic neurons without inflammation by microglial triggering receptor expressed on myeloid cells-2. J Exp Med 2005;201:647-657.

127. Colonna M. TREMs in the immune system and beyond. Nat Rev Immunol 2003;3:445-453.

128. Peng Q, Malhotra S, Torchia JA, Kerr WG, Coggeshall KM, Humphrey MB. TREM2- and DAP12-dependent activation of PI3K requires DAP10 and is inhibited by SHIP1. Sci Signal 2010;3:ra38.

129. Melchior B, Garcia AE, Hsiung BK, et al. Dual induction of TREM2 and tolerance-related transcript, Tmem176b, in amyloid transgenic mice: Implications for vaccine-based therapies for Alzheimer's disease. ASN Neuro 2010;2:e00037.

130. N'Diaye EN, Branda CS, Branda SS, et al. TREM-2 (triggering receptor expressed on myeloid cells 2 ) is a phagocytic receptor for bacteria. J Cell Biol 2009;184:215-223.

131. Neumann H, Takahashi K. Essential role of the microglial triggering receptor expressed on myeloid cells-2 (TREM2) for central nervous tissue immune homeostasis. J Neuroimmunol 2007;184:92-99.

132. Bouchon A, Hernandez-Munain C, Cella M, Colonna M. A DAP12-mediated pathway regulates expression of CC chemokine receptor 7 and maturation of human dendritic cells. J Exp Med 2001;194:1111-1122.

133. Frank S, Burbach GJ, Bonin M, et al. TREM2 is upregulated in amyloid plaque-associated microglia in aged APP23 transgenic mice. Glia 2008;56:1438-1447.

134. Jonsson T, Stefansson H, Steinberg S, et al. Variant of TREM2 associated with the risk of Alzheimer's disease. N Engl J Med. 2013;368:107-116.

135. Guerreiro R, Wojtas A, Bras J, et al. TREM2 variants in Alzheimer's disease. N Engl J Med 2013;368:117-127.

136. Numasawa Y, Yamaura C, Ishihara S, et al. Nasu-hakola disease with a splicing mutation of TREM2 in a Japanese family. Eur $\mathrm{J}$ Neurol 2011;18:1179-1183.

137. Borroni B, Ferrari F, Galimberti D, et al. Heterozygous TREM2 mutations in frontotemporal dementia. Neurobiol Aging 2014;35: 934.e7-934.10.

138. Rayaprolu S, Mullen B, Baker M, et al. TREM2 in neurodegeneration: Evidence for association of the p.R $47 \mathrm{H}$ variant with frontotemporal dementia and Parkinson's disease. Mol Neurodegener 2013;8:19-1326-8-19.

139. Cady J, Koval ED, Benitez BA, et al. TREM2 variant p.R47H as a risk factor for sporadic amyotrophic lateral sclerosis. JAMA Neurol 2014;71:449-453.

140. Jiang T, Yu JT, Zhu XC, Tan L. TREM2 in Alzheimer's disease. Mol Neurobiol 2013;48:180-185. 
141. Jiang T, Tan L, Zhu XC, et al. Upregulation of TREM2 ameliorates neuropathology and rescues spatial cognitive impairment in a transgenic mouse model of Alzheimer's disease. Neuropsychopharmacology 2014 Jul 3.

142. Ulrich JD, Finn MB, Wang Y, et al. Altered microglial response to abeta plaques in APPPS1-21 mice heterozygous for TREM2. Mol Neurodegener 2014;9:20-1326-9-20.

143. Sieber MW, Jaenisch N, Brehm M, et al. Attenuated inflammatory response in triggering receptor expressed on myeloid cells 2 (TREM2) knock-out mice following stroke. PLoS One 2013;8:e52982.

144. Piccio L, Buonsanti C, Mariani M, et al. Blockade of TREM-2 exacerbates experimental autoimmune encephalomyelitis. Eur J Immunol 2007;37:1290-1301.

145. Streit WJ, Xue QS. Life and death of microglia. J Neuroimmune Pharmacol 2009;4:371-379.

146. Mosher KI, Wyss-Coray T. Microglial dysfunction in brain aging and Alzheimer's disease. Biochem Pharmacol 2014;88:594-604.

147. Mildner A, Schmidt H, Nitsche M, et al. Microglia in the adult brain arise from ly-6ChiCCR2+ monocytes only under defined host conditions. Nat Neurosci 2007;10:1544-1553.

148. Ajami B, Bennett JL, Krieger C, Tetzlaff W, Rossi FM. Local self-renewal can sustain CNS microglia maintenance and function throughout adult life. Nat Neurosci 2007;10: $1538-1543$.

149. Malm TM, Koistinaho M, Parepalo M, et al. Bone-marrow-derived cells contribute to the recruitment of microglial cells in response to beta-amyloid deposition in APP/PS1 double transgenic Alzheimer mice. Neurobiol Dis 2005;18:134-142.

150. Simard AR, Soulet D, Gowing G, Julien JP, Rivest S. Bone marrowderived microglia play a critical role in restricting senile plaque formation in Alzheimer's disease. Neuron 2006;49:489-502.

151. Naert G, Rivest S. The role of microglial cell subsets in Alzheimer's disease. Curr Alzheimer Res 2011;8:151-155.

152. Gordon S, Taylor PR. Monocyte and macrophage heterogeneity. Nat Rev Immunol 2005;5:953-964.

153. Sunderkotter C, Nikolic T, Dillon MJ, et al. Subpopulations of mouse blood monocytes differ in maturation stage and inflammatory response. J Immunol 2004;172:4410-4417.

154. Selenica ML, Alvarez JA, Nash KR, et al. Diverse activation of microglia by chemokine (C-C motif) ligand 2 overexpression in brain. J Neuroinflammation 2013;10:86-2094-10-86.

155. Butovsky O, Jedrychowski MP, Moore CS, et al. Identification of a unique TGF-beta-dependent molecular and functional signature in microglia. Nat Neurosci 2014;17:131-143. 